\title{
Watuchi. Enigmas y saberes infantiles en los Andes del sur del Perú
}

Watuchi. Énigmes et savoirs infantiles dans les Andes du sud du Pérou

Watuchi. Riddles and children's skills in the South Peruvian Andes

\section{Palmira La Riva González}

\section{(2) OpenEdition}

Journals

\section{Edición electrónica}

URL: http://journals.openedition.org/bifea/4147

DOI: $10.4000 /$ bifea.4147

ISSN: 2076-5827

\section{Editor}

Institut Français d'Études Andines

Edición impresa

Fecha de publicación: 1 diciembre 2013

Paginación: 369-388

ISSN: 0303-7495

Referencia electrónica

Palmira La Riva González, « Watuchi. Enigmas y saberes infantiles en los Andes del sur del Perú », Bulletin de l'Institut français d'études andines [En línea], 42 (3) | 2013, Publicado el 08 diciembre 2013, consultado el 05 noviembre 2020. URL : http://journals.openedition.org/bifea/4147 ; DOI : https:// doi.org/10.4000/bifea.4147

\section{(c)}

Les contenus du Bulletin de l'Institut français d'études andines sont mis à disposition selon les termes de la licence Creative Commons Attribution - Pas d'Utilisation Commerciale - Pas de Modification 4.0 International. 


\title{
Watuchi. Enigmas y saberes infantiles en los Andes del sur del Perú
}

\author{
Palmira La Riva González*
}

\section{Resumen}

Este artículo se interesa en la realización de adivinanzas como evento de habla al que se inician los niños desde antes de los 5 años en dos comunidades campesinas del departamento de Cuzco; Surimana (provincia de Canas) y Ccachin (provincia de Calca). Estas adivinanzas forman parte de un repertorio común compartido por todos los niños de la comunidad. Sin embargo, cada niño puede enriquecer este repertorio por la creación personal. El término quechua utilizado para referirse a las adivinanzas es watuchi. Este vocablo deriva del verbo watu- «adivinar» o «presentir». El aprendizaje de las adivinanzas forma parte de un conjunto de prácticas semióticas más amplio que aprenden los niños desde la primera infancia. Se consideran también la correlación entre los juegos verbales, el desarrollo de las capacidades cognitivas y la socialización de los niños.

Palabras clave: Andes, Perú, primera infancia, juegos verbales, aprendizaje, capacidades cognitivas, socialización

\section{Watuchi. Énigmes et savoirs infantiles dans les Andes du sud du Pérou}

\section{Résumé}

Cet article s'intéresse à la performance de devinettes comme acte de parole auquel s'initient les enfants de moins de 5 ans dans deux communautés paysannes du département de Cuzco : Surimana (province de Canas) et Ccachin (province de Calca). Ces devinettes appartiennent à un répertoire commun partagé par tous les enfants de la communauté. Cependant, chaque enfant peut enrichir ce répertoire par sa création personnelle. Watuchi est le terme quechua qui désigne les devinettes. Ce vocable dérive du verbe watu- « deviner » ou « pressentir ». L'apprentissage des devinettes s'insère dans 
un ensemble de pratiques sémiotiques plus large que les enfants maîtrisent dès la petite enfance. Les corrélations entre les jeux de mots, le développement des capacités cognitives et la socialisation des enfants sont également prises en compte.

Mots-clés : Andes, Pérou, petite enfance, jeux de langage, apprentissage, capacités cognitives, socialisation

\title{
Watuchi. Riddles and children's skills in the South Peruvian Andes
}

\begin{abstract}
This article focuses on the performance of riddles as speech events that learn children under 5 years old in two rural communities of the department of Cuzco: Surimana (province of Canas) and community Ccachin (province of Calca). These riddles belong to a common directory shared by all children of the community. However, each child can enrich this directory through his own creation. Watuchi is the term referring to Quechua riddles. Its derives from the verb watu "to guess" or "to have an intuition". Learning riddles is part of a vast set of semiotic practices managed by children since early childhood. Correlations between speech acts, cognitive skills development and children' socialization are also discussed.
\end{abstract}

Keywords: Andes, Peru, early childhood, speech acts, learning, cognitive skills, socialization

«Rien de définitif ne s'est encore produit au monde, le denier mot du monde et sur le monde n'a pas encore été dit,

le monde est ouvert et libre...»

(Bakhtine, 1970 : 195)

\section{INTRODUCCIÓN}

Si bien es cierto que los niños y niñas han siempre ejercido, en mayor o menor grado, su capacidad de acción y de respuesta al medio en el que se desenvuelven, el enfoque que considera a los niños como actores sociales y valoriza sus propios discursos es reciente en las ciencias sociales (Delalande, 2009; Sirota, 2006; 2012; Bergonnier-Dupuy, 2005). En este artículo nos inscribimos en esta misma perspectiva y nos interesaremos así en la realización de adivinanzas como juegos verbales al que se inician los infantes menores de cinco años. Estas adivinanzas forman parte de un repertorio común en las que participa toda la comunidad, quedando entendido que cada niño puede crear su propio repertorio en función de sus capacidades creativas. Proceso esencial en el ciclo de vida del niño, la adquisición de la lengua y la realización de juegos verbales ocupan un rol preponderante en el desarrollo de las capacidades cognitivas, así como en el proceso de socialización entre pares y adultos. El objetivo de este artículo es 
iniciar una reflexión sobre estos aspectos, poco estudiados hasta ahora. En este texto veremos cómo la adquisición de las habilidades lingüísticas que supone la realización de estos juegos de lenguaje está relacionada con las modificaciones ontológicas en relación con el ritual del primer corte de pelo (rutuchikuy) que abordaremos en una primera instancia para luego considerar las concepciones relativas a la adquisición de la lengua vinculada con la adhesión del principio vital (animu) al cuerpo. En el acápite subsiguiente nos concentraremos en las realizaciones lingüísticas de los niños de forma general y en un contexto de habla quechua de manera específica, tomando en cuenta la relación entre la lengua y las representaciones locales relativas a la adquisición de los saberes y conocimientos. Enseguida nos centraremos en el estudio de las adivinanzas, considerando las funciones tanto cognitivas como sociales de las mismas. Así el último acápite tratará de la función de socialización de los acertijos.

Las informaciones utilizadas en este artículo provienen de un largo trabajo de campo en el marco de una tesis doctoral sobre las representaciones del cuerpo en la comunidad de Surimana (provincia de Canas en el departamento del Cuzco). La obtención de este material es el resultado de la convivencia diaria en la comunidad y en la recopilación de adivinanzas. Algunas de ellas provienen también de la comunidad de Ccachin (provincia de Calca en el departamento de Cuzco).

\section{RUTUCHIKUY. EL PRIMER CORTE DE PELO Y EL DESARROLLO DE LAS CAPACIDADES COGNITIVAS}

En este artículo no consideramos la dimensión social y simbólica de este rito, que entrena un complejo parentesco ritual analizado en profundidad por otros autores (Christinat, 1989; Suremain, 2010). Nos limitamos en señalar que en el largo proceso de construcción cultural del cuerpo-persona mediante los ritos de paso, que marcan no solo el cambio de estatus del individuo en el seno de la sociedad sino que entrenan también modificaciones ontológicas irreversibles, el primer corte de pelo en la comunidad quechua hablante de Surimana (Cuzco) juega un rol primordial. Con este ritual, que se realiza hacia los 3 o 4 años de edad, pareciera llevarse a cabo la «domesticación» del animu (principio vital) y una mayor adhesión del mismo al cuerpo, desarrollándose así las capacidades cognitivas del niño, entre las que podemos mencionar la facultad de soñar1 y

1 En los Andes la experiencia onírica constituye una fuente esencial de la adquisición de saberes tanto para los especialistas rituales como para la población en general. Para un estudio sobre la relación entre los sueños «oraculares» y los ritos de iniciación de los especialistas rituales véase Polia Meconi (1994); Glass-Coffin (2003: 77) y Fernández Juárez (1995a: 215; 1995b). En la zona del Ausangate (Cuzco) Ricard Lanata registra un ejemplo en el que el especialista ritual accede al saber terapéutico por medio de un sueño. En este caso específico, es la divinidad de la montaña (Apu) quien transmite el saber al que sueña (Ricard Lanata, 2010: 104, 105). Para los laymi (Bolivia) Olivia Harris señala que las sirenas transmiten las melodías de los instrumentos a los músicos por medio de los sueños: the tune is communicated either an a dream to the owner of the instrument, or directly through the instrument which plays in a new way (Harris, 1980: 83). 
el desarrollo de las competencias lingüísticas. Estas directamente asociadas a la facultad de la razón, como de la conciencia (yuyay), confiriendo al niño su status plenamente humano. Digamos además, que por medio de esta ceremonia se refuerzan también las diferencias de género, puesto que después de este ritual, las wawa (bebés) abandonan la phalika (vestimenta unisex) para vestirse de manera diferenciada, como niños y niñas así como asumir una serie de actividades asociadas a su género.

\section{YUYAY HAP'ISQA. SEMBRANDO Y FIJANDO LAS PALABRAS}

Según la expresión de mis interlocutores, la adquisición de las habilidades lingüísticas es posible a partir del momento en que el niño está «despierto». Estas habilidades están ligadas a la posesión de la razón y de la conciencia (yuyay) lo que se expresa con la frase yuyay hap'isqa. El verbo usado para hacer referencia a esta capacidad cognitiva es yuya- «razonar», «pensar», «recordar». Es así que el verbo substantivado yuyay significa a la vez memoria, inteligencia, conciencia y razón (Itier, 2011: 250; Academia Mayor de la Lengua Quechua, 2005: 771).

Según las representaciones del cuerpo-persona vigentes en la comunidad, dos centros corporales aparecen como las sedes principales de estas funciones: la fontanela (uma pukyu) y el corazón (sunqu)2. En Surimana se dice que la fontanela es el lugar de la memoria y del origen de las palabras. La importancia de esta parte del cuerpo en la adquisición del lenguaje articulado es mencionada también por Arnold \& Yapita para los Andes de Bolivia (2002: 62). En efecto, en el ayllu Qaqachaka (Oruro), la fontanela se denomina en aimara palabr p uq antiriwa «sitio de la palabra», literalmente «reproductor de palabras» (Arnold \& Yapita, 1996: 321). Considerada también como «un campo que uno siembra», la adquisición de conocimientos en la fontanela se expresa con el verbo tarpu-sembrar, meter al interior, introducir, y los saberes desarrollados son vistos como la reproducción de vegetales:

Chay uma pukyuqa sirvin pinsanapaq, napaq... chay pinsanapaq, akna imapis ruwanaykipis anchaypaq sirvin riki umaman riki tarpushanchis imatapis riki.

La fontanela sirve para pensar, pensar en todo lo que debes hacer, para eso sirve la cabeza; en la cabeza sembramos todo lo que uno quiere (Abelino Huillka Huaraya).

El sunqu aparece también como centro privilegiado de la elaboración de la memoria y del pensamiento. Es así que la misma expresión con el verbo tarpu- se utiliza en el caso del sunqu en cuanto receptáculo de conocimientos: sunquypi tarpushani. «Lo imprimo (grabo) en mi espíritu» (Itier, 2011: 211).

2 El termino «corazón» no traduce la entidad corporal sunqu en todas sus dimensiones, órgano vital, sede de las funciones digestivas, de la memoria y cognitivas. 
Realizada en el sunqu o en la fontanela (en la cabeza de manera general), la actividad cognitiva a la que el término yuya- hace referencia, está asociada a la presencia efectiva del animu en el cuerpo, pues se dice de un niño que sufre el síndrome del «susto» (mancharisqa ) es decir, que ha perdido su animu, que ya no tiene ni entendimiento ni razón, que por el susto pierde «su pensamiento» y «la capacidad de pensar», según la expresión de mis interlocutores. En el testimonio siguiente, la relación de interdependencia entre el sunqu, la capacidad intelectual expresada con el termino yuya-, y el animu es explicita:

Sunqun mana allinchu kashan. Yuyaynin mana kashanchu chay wawaq, debil kashan. Maypachachus chay animuta waqyanki chayraq riki chay wawa allin kashan riki.

Su corazón va mal. No tiene conciencia (ya no piensa), está débil, frágil. Pero cuando tú llamas a su alma, está bien (Natividad Cuebas).

La ausencia del animu en el cuerpo da cuenta también de la condición de las personas sordo-mudas, «idiotas», quienes se supone han perdido su animu, lo que puede además provocar la locura, como se advierte en el presente testimonio:

Waq'ayarunku, ... mancharisqamanta, animun lluqsiruqtin, anchay waq'a kan riki.

"A causa del miedo se vuelven locos, es porque el alma se va que hay locos" (Natividad Cuebas).

Es así que la presencia del animu garantiza tanto la razón como el equilibrio térmico de las substancias corporales implicadas en el mantenimiento de la salud mental y corporal, dentro del sistema humoral sobre la base de las representaciones del cuerpo-persona en esta comunidad y en los Andes en general (La Riva González, 2005: 2012).

En Surimana, como en otras regiones de los Andes, el desarrollo de las capacidades lingüísticas define el estatus de humano (runa) y el de la madurez, mientras que la ausencia de lenguaje y el silencio se asocian a la barbarie o a una palabra defectuosa, a lo «no suficientemente cocido» y a la muerte. De esta forma, el termino kapka «semi-cocido» puede significar también «mala pronunciación, palabra defectuosa», vehiculando una connotación negativa (Lira, 1982: 116). Esta representación es compartida por los laymi (Bolivia) quienes consideran que la adquisición del lenguaje y el desarrollo de las competencias lingüísticas es lo que confiere plenamente el estatus de humano (Harris, 1980: 72-73). De manera significativa, la experticia comunicativa caracteriza a los especialistas rituales de más alto rango (altumisayuq), capaces de comunicar con las divinidades tutelares (Fernández Juárez, 1997: 69; Ricard Lanata, 2010).

\section{JUEGOS DE LENGUAJE, LENGUAJE DE LOS NIÑOS...}

Hasta muy recientemente, el lenguaje infantil no ha retenido la atención de los antropólogos, quienes con más frecuencia se han concentrado en las prácticas discursivas de los adultos: mitos, leyendas, rituales, considerados como más 
dignos de interés. No obstante, debemos mencionar los estudios africanistas que constituyen una excepción en este desinterés. En efecto, en lo que concierne el lenguaje de los niños, los antropólogos y lingüistas, especialistas de las tradiciones orales en África se han preocupado por los juegos verbales y rutinas conversacionales entre niños y adultos, resaltando la función pragmática y cognitiva de los mismos (Calame-Griaule, 1963; 1970; Rabain, 2003: 60). Varios autores han señalado que las diversas prácticas lingüísticas infantiles (adivinanzas, juegos de palabras, cuentos, canciones, trabalenguas) en tanto que juegos verbales, contribuyen al desarrollo de las capacidades cognitivas así como a la socialización de los niños.

El término de «juegos de lenguaje» es empleado aquí como práctica discursiva en el sentido de «juegos infantiles que acompañan el aprendizaje de la lengua», como en el sentido más general que le da Wittgenstein en su segunda filosofía:

yo llamaría también «juego de lenguaje» al conjunto formado por el leguaje y las actividades con las cuales se entrelaza» (...) «la expresión juego de lenguaje hace referencia a que hablar una lengua forma parte de una actividad o de una forma de vida (Wittgenstein, 2005: § 7 y $§ 23$ ).

Siguiendo los postulados de la etnografía de la comunicación sobre los fenómenos lingüísticos, estos juegos verbales son considerados como actos sociales que se insertan dentro de un marco de prácticas semióticas más amplio que exige tomar en cuenta la dimensión social y pragmática de la lengua, así como una exploración sistemática de «situaciones y usos, estructuras y funciones de la palabra, considerada como una actividad de pleno derecho» (Hymes, 1964). Esta concepción, que toma en cuenta el rol de la palabra en la vida social en cuanto forma integrante de la cultura, se opone a la herencia lingüística estructural, construida en base a la distinción entre lengua y palabra, y que por lo tanto no toma en cuenta las funciones sociales de los actos lingüísticos (Volochinov/Bakhtine, 1981 [1926]; Bourdieu, 1982; Lindenfeld, 1984). Considerar los aspectos sociales de los juegos de lenguaje (en este caso de las adivinanzas) es tanto más necesario que estas cumplen un rol fundamental en el proceso de socialización de los niños.

\section{LAS ADIVINANZAS: ENIGMAS Y FUENTE DE SABERES}

La realización de las adivinanzas como práctica lingüística ha suscitado el interés de los lingüistas (Hamnett, 1967: 380; Jakobson, 1977; Todorov, 1978; Faïk Nzuji, 1976) quienes las han analizado en su aspecto formal y lingüístico, pero sin necesariamente tomar en cuenta el contexto cultural y social en el que se realizan (Hamnett, 1967: 380). No obstante, numerosos autores han señalado la importancia de las funciones y del rol que juegan las adivinanzas en el desarrollo de las capacidades de categorización y de los procesos cognitivos en general (Freud, 1983 [1905]; Hamnett, 1967; Williams, 1963). En relación a estas competencias, la lingüista Faïk-Nzuli refiriéndose a las adivinanzas tonales luba (África central) señala que el término utilizado en esta lengua para hacer referencia a las adivinanzas significa «palabra cuyo desciframiento exige la inteligencia, la perspicacidad y una agilidad de la mente poco comunes» (Faïk-Nzuli, 1976: 21). 
Roger Bastide, por su parte, resalta el carácter específico de las adivinanzas en los siguientes términos:

Lo esencial de la adivinanza consiste en reunir por lo menos en una sola unidad lingüística dos sentidos que teóricamente pertenecen a dos niveles de significación (o de clasificación) diferentes pero que hacen corto circuito en esta unidad lingüística (Bastide, 1970: 958).

Este «corto circuito» del pensamiento del que habla Bastide implica el despliegue de la lógica simbólica que las adivinanzas comparten con los mitos y que radica en «una manera de pensar que consiste, sin negar el orden de la clasificación, en avanzar por medio de la analogía de un plan de la realidad a otro» (Bastide, 1970: 995). Esta manera de pensar de la que habla Bastide se caracteriza por la ambivalencia, ambigüedad y polisemia. Es así que el pensamiento analógico con la complejidad heurística que lo caracteriza confiere a la práctica de las adivinanzas una función cognitiva indiscutible. La elaboración y ejecución de las adivinanzas permite de esta manera la transmisión de distintos tipos de saberes que se articulan en un juego de correspondencias dinámicas como veremos en algunos ejemplos más adelante.

\section{ADIVINANDO EN LOS ANDES}

En lo que se refiere a los Andes, Isbell \& Roncalla (1977)3 han señalado la importancia de los acertijos en el desarrollo cognitivo de niños y adolescentes quechuahablantes mediante la comprensión y el desciframiento de las metáforas empleadas en estos juegos verbales. Estos autores recuerdan que «las metáforas [...] desempeñan un rol esencial en el desarrollo cognitivo y semántico en los niños quechuahablantes que se entrenan en la elaboración de adivinanzas» (Isbell \& Roncalla, 1977: 20). En Surimana (provincia de Canas) y en la comunidad de Ccachin (provincia de Calca), las adivinanzas tienen un rol fundamental, no solamente en el desarrollo de las capacidades cognitivas sino también en la socialización de los niños. Estos se inician desde cinco o seis años a la realización de este género de la literatura oral, disponiendo de un repertorio bastante amplio que divulgan con entusiasmo. Estas adivinanzas pertenecen a un repertorio común compartido por toda la comunidad, pero en el cual cada niño puede improvisar un repertorio personal con relativa libertad creativa.

El término quechua empleado para designar el juego de las adivinanzas es watuchi, asociado al término watu- «adivinar», «presentir» con el causativo o factivo -chi-. El mismo término se emplea para hablar de «presagios», de «pronósticos» o de

3 En efecto, para la región andina los estudios sobre las adivinanzas, a excepción del clásico estudio de Isbell \& Roncalla, y los estudios más recientes de Virginia Zavala $(2001$; 2006) que dan luces sobre la importancia de las adivinanzas, se limitan a una lista de adivinanzas sin integrarlas a un sistema semiótico más vasto. Véase Cáceres Vargas (1996), Kleymeyer (1993); Altamirano (1984) y Navarro del Águila (1947). 
«predicciones» (Academia mayor de la lengua quechua, 2005: 731). En su forma watupaku-, este vocablo está asociado a la esfera de los «presentimientos», de la «intuición» y de la «sospecha» y aparece como sinónimo de musya- (Itier, 2011: 239) 4 y, en este caso, «percibido», «sentido» en el sunqu: «no tengo ganas de comer, perdí el apetito, qué será lo que mi corazón trata de adivinar?» (Gina Maldonado). Es importante señalar que las personas que practican la adivinación (con las hojas de coca, con barajas, o con los libros de San Ciprián, patrón de los brujos) son llamados -watuq. En este sentido, el aprendizaje del repertorio de las adivinanzas existentes, así como la elaboración personal de las mismas, forman parte del proceso de socialización y del desarrollo lingüístico del niño, así como el de las actividades relativas a la adivinación en un sentido amplio. Entre estas prácticas mánticas podemos mencionar tanto el sistema de pronóstico agrícola y meteorológico de origen europeo, basado en la observación de indicadores climáticos de los primeros diez días del mes de agosto conocido como «cabañuelas», la interpretación del «comportamiento de las nubes» para determinar al vencedor en el chiaraje (batalla ritual) por ejemplo, así como la lectura de hojas de coca y las prácticas adivinatorias empleadas en los procedimientos terapéuticos 5 . Estas prácticas, de las cuales los sueños forman parte, juegan un rol fundamental en la vida cotidiana de las poblaciones andinas. Cabe señalar que los procedimientos cognitivos utilizados en la descodificación o interpretación de los sueños son los mismos que se utilizan para descifrar las adivinanzas. Estas se insertan así dentro de un conjunto de prácticas semióticas mucho más amplio, al cual se inician los niños desde la primera infancia. Estos juegos verbales activan además una serie de capacidades cognitivas que habría que relacionar con los diferentes modos de conocimiento. Algunas de estas complejas correlaciones, así como sus respectivas realizaciones corporales han sido documentadas por González Holguín en el siglo XVII6. Mencionemos al respecto dos de estos modos de cognición/existencia: uno, a través de los sentidos, la percepción y las emociones que se produce directamente en el sunqu:

Sonccoypichuraykuni ñan hapicuni «Entender percibiendo»

Yachacuni yanaycuni «exercitar, o probar, o hazer experiencia»

El otro, por inferencia y análisis del discurso, como en el caso de las adivinanzas y que está ligado al verbo yuya- situado también en el sunqu.

Huatuni «Adivinar algo, o conjeturar, o sacar por discurso, o conjeturas prouidamente» (González Holguín, [1608] 1952: 189).

4 «musya- (CHAN) sentir, presentir, adivinar, sospechar» (Itier, 2011: 144).

5 Entre las técnicas más utilizadas en la zona de estudio, mencionemos la lectura del «huevo», la de las orinas y de las hojas de coca para establecer el diagnostico y definir la terapia adecuada. Para otras zonas andinas podemos mencionar la lectura de las vísceras del cuy.

6 Respecto a las distintas formas de conocimiento, González Holguín hace referencia a los sentidos, al entendimiento, a la intuición o conjetura, a la planificación y a la adivinación: (hamurpayay, unanchay huatuy, yachacuy) (cf. González Holguín, ([1608] 1952: 147, 148, 189, 457, 507, 508, $668,362)$. 
Este término huatuni debe analizarse en relación con los términos hamurpayani, vnanchani «entender con discurso, y razones» (González Holguín, [1608] 1952: 507).

Hamurpayani «entender, o comprender, o considerar hondamente»

Hamutani «conjeturar, sacar por discurso lo que será bueno y sucederá bien y lo que no»

Hamutac «adivinador prudente»

Huatuni «adivinar algo, o conjeturar, o sacar por discurso, o conjeturas prouidamente»

Hamurpayani. Huatuni huatuycachani «Conjeturar»

Hamuttani vnanchani «Entender»

Hamuttak, o vnanchasoncco o hamuttana o vnanchana «Entendimiento»

Hamuttay, y vnanchay «Entendimiento por intelección o acto de entender» Hamurpayani, vnanchani «Entender con discurso y razones»

Para este mismo período colonial, un testimonio de fray Martín de Murúa pone en relación directa las adivinanzas y el acto de adivinar y de filosofar:

y tenían juntamente estos Ingas unos médicos o filósofos adivinos que se dicen Guacácue, los cuales andaban desnudos por los lugares más apartados y sombríos desta región, y por esta razón se llamaban así; andando solos por los desiertos, sin reposo ni sosiego se daban a la adivinanza o filosofía (Murúa, [1590] 1946: 155-156).

Si bien en las definiciones existentes hoy en día del término watuchi no encontramos glosas que se refieran a la «comprensión mediante el análisis del discurso» (cf. González Holguín) como para el siglo XVII, no es menos cierto que la elaboración y el desciframiento de los enigmas planteados en las adivinanzas, exigen por parte de los niños el desarrollo del razonamiento analógico en la descodificación de metáforas y metonimias. Estas son, como lo subrayan Lakoff \& Johnson (1985), mucho más que un procedimiento de la imaginación poética o de la «ornamentación retórica», la metáfora sería el fundamento mismo de «nuestro sistema conceptual» y está presente también en «el pensamiento y la acción» (Lakoff \& Johnson, 1985).

En el estudio sobre las adivinanzas en lengua quechua realizado por Isbell \& Roncalla (1977), estos autores analizan las relaciones entre el lenguaje figurativo, empleado en las adivinanzas, y los diferentes niveles de desarrollo cognitivo; desde lo más concreto (ritmo) hasta lo más abstracto (conceptos):

las adivinanzas en lengua quechua (las más simples) comienzan con la percepción de sonidos (ritmo) y se desarrollan hacia conocimientos más abstractos sobre el movimiento [...] la comprensión de metáforas [...], las proposiciones lógicas, etc. (Isbell \& Roncalla, 1977: 45-47).

Si bien estos autores sitúan la iniciación a las adivinanzas (onset of ridling) y el desarrollo de las capacidades cognitivas antes mencionadas, hacia los 11 y 12 años, e incluso hasta la edad de contraer matrimonio (Isbell \& Roncalla, 1977: 21 - 
22), en Surimana y en otras comunidades, Yargha Kunka (provincia de Acomayo), Ccachin, hemos podido observar que los niños se inician desde temprana edad (5 o 6 años), a esta práctica lingüística como lo hemos señalado previamente.

En el contexto estudiado por Isbell y Roncalla, es muy probable que la ausencia de la práctica de adivinanzas entre los niños más pequeños se deba más bien al resultado de las relaciones de la práctica de trabajo de campo y a la orientación de su investigación, tal como ellos mismos lo mencionan:

no logramos recoger adivinanzas entre los niños más jóvenes. Hay que decir, sin embargo, que nunca participamos a las actividades lúdicas de los mas pequeños entre los cuales estas actividades son susceptibles de realizarse (Isbell \& Roncalla, 1977: 21-22).

En lo que concierne las operaciones y competencias implícitas en la resolución y elaboración libre de las adivinanzas de las que hemos hablado más arriba, en este artículo nos contentamos con una aproximación sucinta de las mismas. Limitémonos a señalar que incluso a la edad de 5 años, los juegos de metáforas, de analogías y ambigüedad que entran en juego en las adivinanzas son bastante complejos y manifiestan una función poética innegable (Jakobson, 1963: 218)7 que los niños dominan bastante bien.

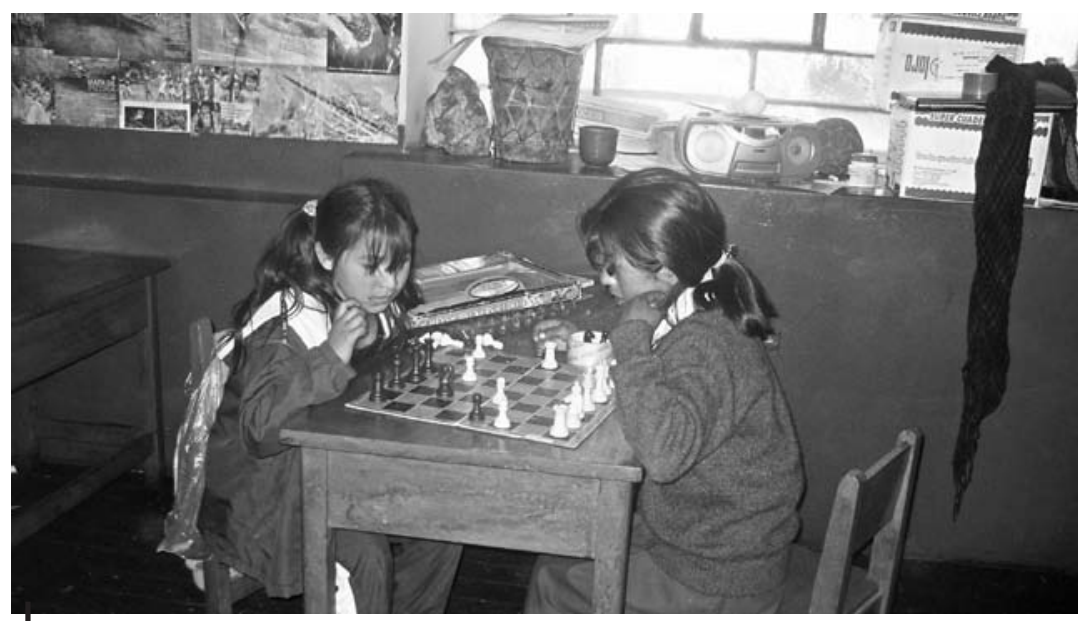

Figura 1 - Niñas jugando en la escuela (comunidad de Ccachin, provincia de Calca)

(C) Palmira La Riva González, 2011

7 Román Jakobson ha señalado esta capacidad poética en los niños en los siguientes términos: «En el niño aparece, desde muy temprana edad, la función [poética]... la atención dirigida hacia el lenguaje, sin relación directa al objeto sobre el que el niño habla. Este es un fenómeno que aparece en el niño a una edad temprana. A veces uno se sorprende de [constatar] hasta que punto el niño se preocupa, no por los fenómenos extralingüísticos, pero por el lenguaje mismo» (Documento INA, Boston, 23 de julio, 1970). 
En lo que se refiere a las adivinanzas en quechua, tal como lo subraya Kleymeyer, se presentan muchos aspectos que destacar en este género de la literatura oral:

el ritmo, el contraste, la onomatopeya, las analogías, el antropomorfismo, el humor, etc. (Kleymeyer, 1993: 15).

Por otra parte, estas adivinanzas implican un gran conocimiento de la vida de la comunidad que incluye los diversos elementos de la naturaleza (animales, plantas, frutas), como lo podemos apreciar en los siguientes acertijos:

Nuqaq sutiymi Javier Puq'u T'aqa, nuqa watuchiykuchata ruwarasaq: imasmari imasmari? Huk maymilli, tawa patapis, iskay laph'ili, huk ch'uñuli, imataq kanmanri?: allqu.

Me llamo Javier Puq'u T'aqa, haré una adivinanza: ¿qué será qué será?

Uno que se mueve, cuatro en patas, dos orejas que cuelgan, una nariz : un perro.

Nuqaq sutiymi Noemi Mayu Waman, imasmari imasmari? altumpi cruzhina chakataran pampaman suyt'uruhina, imataq kanmanri?: condor.

Me llamo Noemí Mayu Waman, ¿qué será qué será? En lo alto crucificado como una cruz y en la pampa como arrodillado: condor.

Nuqaq sutiymi Carmelo Quispe, nuqa watuychiykuchata ruwasaq : hawan teja ukhun kalamina, imataq kanmanri? = k'allampa

Me Ilamo Carmelo Quispe, haré una adivinanza: por fuera teja, por dentro calamina, ¿qué será qué será?: kallampa (hongo)

Nuqaq sutiymi Maria Helena Zuniqa Cruz, nuqa wak watuchiykuchata ruwaramusaq: huk muquchapi piñachakuq tiyashan imataq kanman? Achupalla8.

Me llamo María Helena Zuñiqa Cruz, elaboraré una adivinanza:

Sobre una loma como piña está sentada: achupalla.

Nuqaq sutiymi Cárdenas Zuñiga nuqa huk watuchiykita ruwaramusaq

Hawan q'umer, ukhun q'illu imataq kanmanri ?

Me llamo Cárdenas Zuñiqa, haré una adivinanza: ¿qué será qué será?

Por fuera verde, por dentro amarillo = zapallo/pera»

Otros acertijos hacen referencia a las técnicas del arte textil o de la música:

Nuqaq sutiymi Noemi Mayu Huaman, nuqa ruwasaq huk watuchikuyta: imasmari imasmari? kunkan k'uchuna, wiksan caballu siki sunkhayuq, imataq kanmanri?: violin.

Me llamo Noemi Mayu Huaman, haré una adivinanza: ¿qué será, qué será? El cuello para cortar, y la barriga con las barbas/bigotes de cerdas de la cola del caballo: violín.

8 Frailejón silvestre de la familia de las Bromeliáceas. 
Encontramos también la expresión de saberes más abstractos como por ejemplo la connotación de fertilidad y de reproducción de movimientos giratorios plasmados en las siguientes adivinanzas9:

Imasmari, imasmari: Imataq muyuspalla, mayuspalla qulqi ganaq: Molino.

Adivina adivinador: Qué es aquello que dando vueltas no más gana dinero. El molino.

Imasmari, imasmari: Imataq muyuspalla, muyuspalla chichuyakuq : Pushka.

Adivina adivinador: ¿Qué es aquello que dando vueltas no más se embaraza? La rueca.

Otro aspecto que nos interesa es el despliegue analógico entre las diferentes partes del cuerpo y otros espacios, geográfico o de los artefactos. A título de ejemplo veamos la analogía entre el ombligo y las lomas, o la del miembro masculino y la flauta quena:

Nuqaq sutiymi Noemi Mayu Huaman nuqa watuchikuychata ruwasaq: q'ara qhatapi rosas tik'acha tik'ashan imataq kanmanri? = puputi

En una ladera pelada están floreciendo rosas.

Qina mana ukhuyuq: pichirro

¿Qué será, qué será? una quena sin huecos; el miembro viril (Kleymeyer, 1993).

Estos procedimientos metafóricos fundados en la analogía entre los términos exigen de parte de los niños competencias cognitivas complejas que deben ser analizadas en relación a las prácticas y representaciones culturales locales y a las distintas formas de conocimiento. En efecto, tal como lo hemos mencionado previamente, la resolución de las adivinanzas implica un modo de conocimiento mediante el análisis del discurso y la conjetura, lo que se manifiesta con el uso corriente del sufijo modal conjetural -chá, que César Itier define en los siguientes términos:

sufijo que indica que quien habla conoce de lo que habla, sea por conjetura o por deducción o suposición, incluso por adivinación. El empleo del conjetural confiere a la frase un grado de probabilidad importante aunque no absoluto (Itier, 1997: 97).

El sufijo -chá sería utilizado cuando la «inferencia se hace por razonamiento» (Faller, 2007)10. Son estas capacidades de razonamiento las que entran en juego

9 Sobre las características de las adivinanzas y la subestimación de sus dimensiones pedagógicas y cognitivas ver la crítica que hace Zavala de su utilización en la escuela en Perú (Zavala, 2001: 29).

10 Digamos que existe en todos los dialectos quechua los sufijos evidenciales que dan cuenta de la fuente de información: «atestiguativo» -mi (-n después de una vocal) fuente directa de información; «reportativo» -sil-s, fuente indirecta de información, el «conjetural o conjeturativo, dubitativo»-chá, que hace referencia a una forma de conocimiento por conjetura, como mencionado más arriba y el «chusina», que hace referencia a la deducción por apariencia (Itier, curso Inalco 2012-2013, Araoz \& Salas, 1993; Cerrón-Palomino, 1987: 266). Sobre los valores de evidencialidad y de modalidad epistémica del conjetural-chá, véase Faller, 2007. 
en la elaboración y resolución de las adivinanzas. Aparece de esta forma una relación estrecha entre las concepciones del desarrollo de las capacidades cognitivas y las formas de aprehensión de la realidad y los marcadores de validación (evidencialidad) de las fuentes de información de la lengua que aún no se ha estudiado. Dicho en otras palabras, los distintos modos de conocimiento y percepción son correlativos a los diferentes tipos de fuentes de información referenciados en los estudios sobre la evidencialidad (cf. fig. 2) que tratan de las diferentes fuentes de conocimiento e información, así como la manera en que estas son marcadas gramáticalmente en la lengua. En efecto, habría que analizar los posibles vínculos entre los diferentes modos de aprehensión y construcción de la realidad (mediante los sentidos, por inferencia, a través de los sueños, por la adivinación, etc.) las teorías del desarrollo cognitivo locales que hacen referencia a la adhesión del animu al cuerpo, a la solidificación de los huesos y a la posesión de la razón yuyay hap'isqa (La Riva González, 2012) y los marcadores de validez gramaticales o evidenciales.

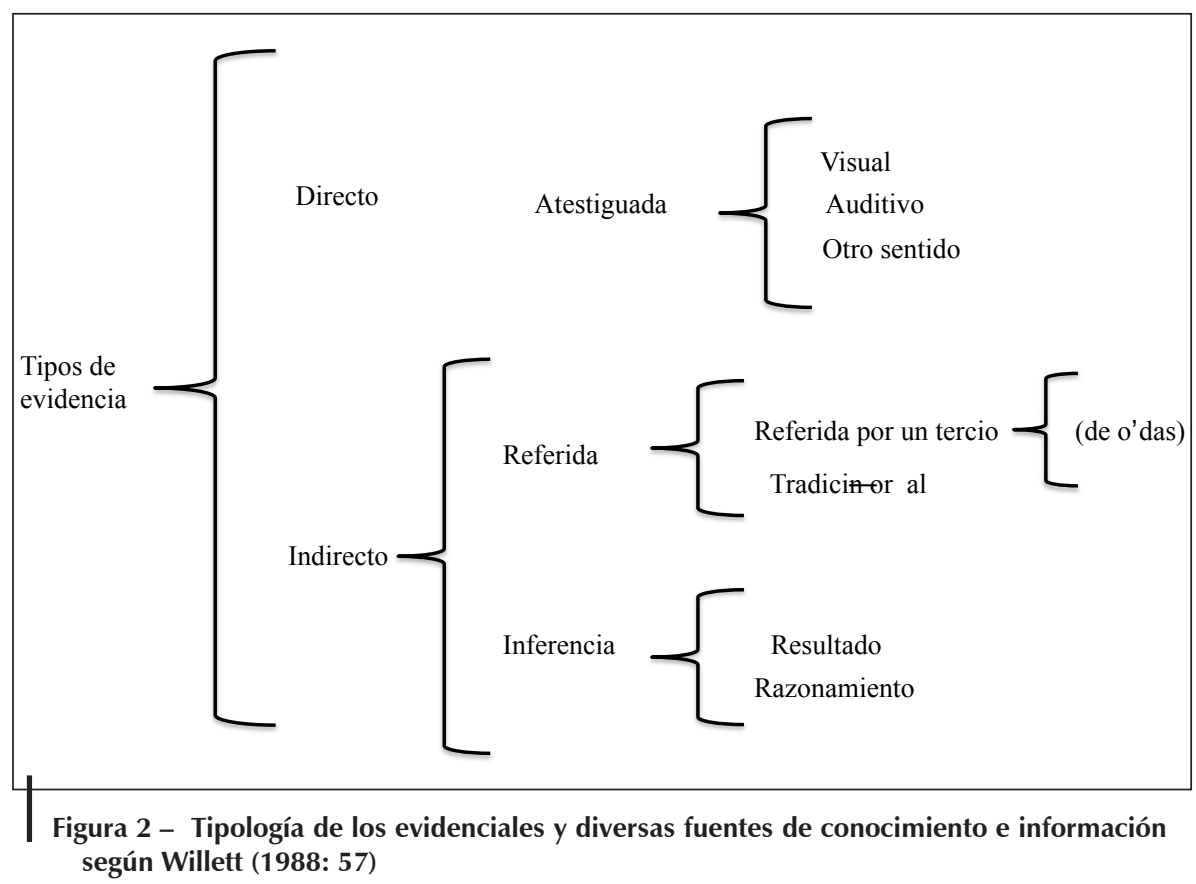

Basadas en la percepción sensorial, por inferencia, por medio de los sueños o mediante los sistemas de adivinación y la tradición oral, las distintas formas de conocimiento y de aprehensión de la realidad presentan realizaciones corporales específicas que habría que analizar a la luz de las representaciones y prácticas corporales andinas. 


\section{LAS ADIVINANZAS Y LA SOCIALIZACIÓN}

Eventos de habla ${ }^{11}$ de un grupo bien definido (en nuestro caso el de 5 a 12 años), las adivinanzas juegan también un rol importante en el proceso de socialización, del desarrollo de la personalidad y de la identidad del niño ${ }^{12}$. En efecto, es sobre todo en su función de socialización que estos juegos de lenguaje - en el sentido que da la etnografía de la comunicación a este concepto (Hymes, 1964)—adquieren todo su valor. Tal como lo subraya Hymes (1964: 215), el evento comunicativo o eventos de habla (speech event) es un instrumento de construcción de la identidad. Digamos además, que si la performance de las adivinanzas constituye un elemento esencial de la construcción de la identidad individual, lo es también de la identidad colectiva. Virginia Zavala reporta que en la zona de Andahuaylas, la performance de adivinanzas funciona «como herramienta de competencia para que una persona pueda defender su identidad como miembro de una comunidad (Zavala, 2006: 134-135). Es así que gran parte del aprendizaje y descubrimientos cognitivos se operan mediante pares y mayores con quienes el niño interactúa en la comunidad.

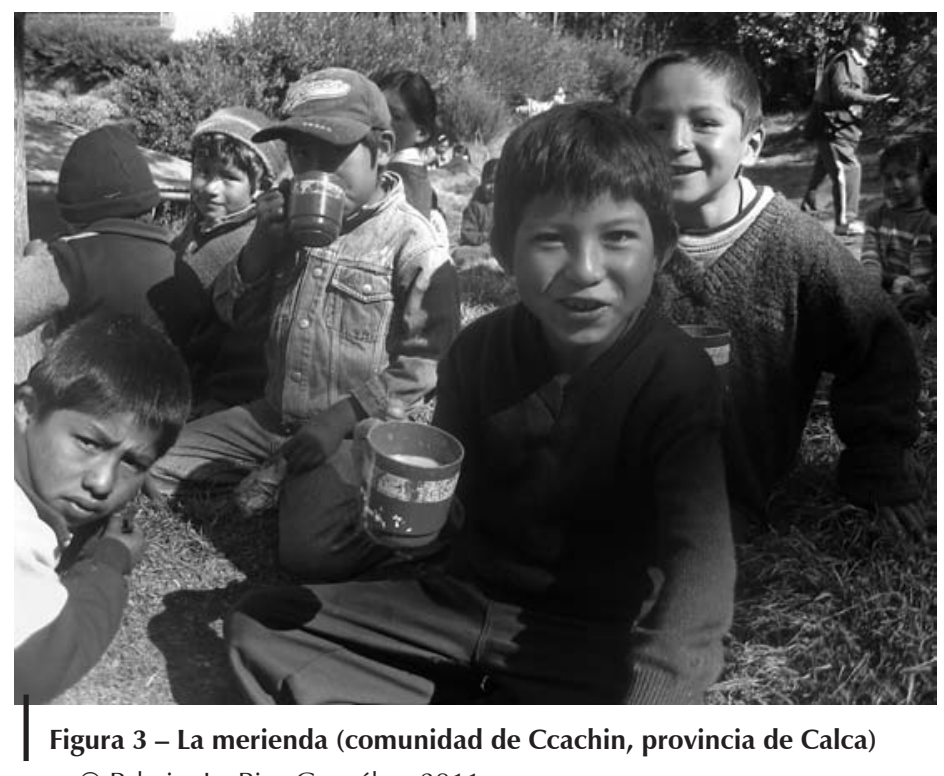

(C) Palmira La Riva González, 2011

11 Empleamos aquí el término «palabra» como dominio linguístico y en el sentido de la etnografía de la comunicación como acto social (Gumperz \& Hymes, 1986 [1972]).

12 Sobre la interrelación entre socialización y evento de habla, véase Hymes (1964: 215): «... the relation of speech event to social interaction and with speech as an instrument for the acquisition and maintenance of personality, role and status»). Véase también Sherzer \& Darnell (1986: 553). Para la relación entre los diferentes «géneros de palabra» y el proceso de socialización ver Robert \& Forman [1972] 1986). 


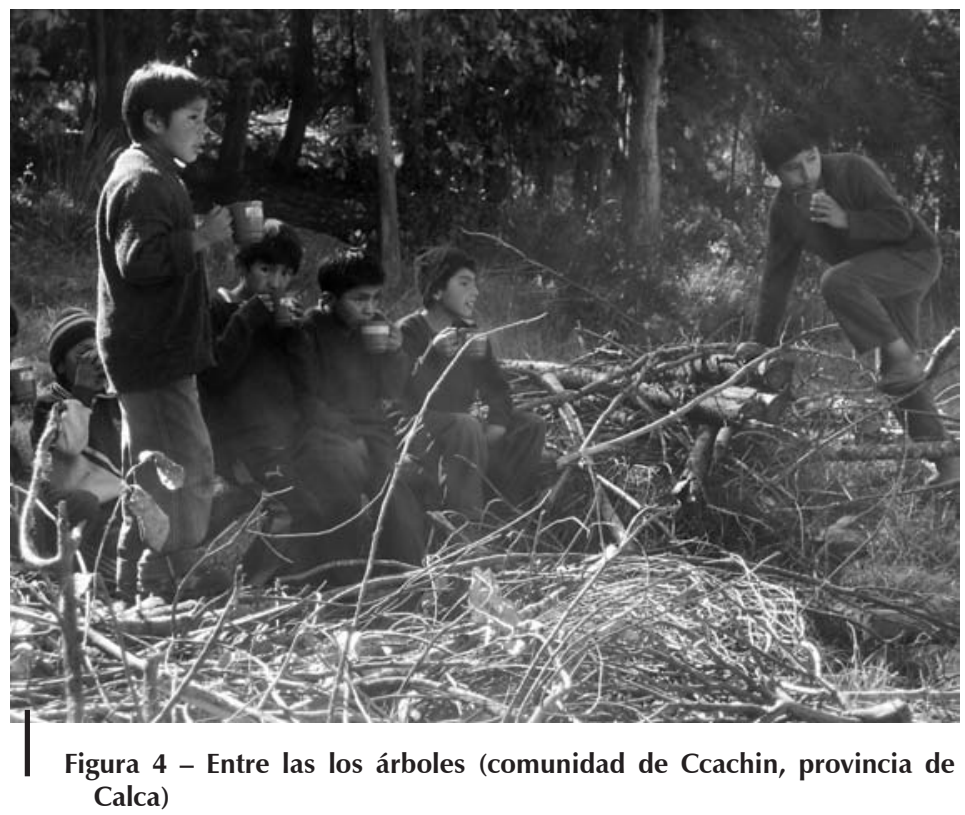

(C) Palmira La Riva González, 2011

En el caso de nuestro estudio, exponemos un contexto de «presentación de símismo» bien preciso, que está en estrecha relación con nuestra intervención en el terreno y con el diálogo establecido con los niños durante nuestra estadía en la comunidad. La elaboración de adivinanzas, canciones o cuentos breves, como parte de la de presentación de la identidad personal era recurrente cada vez que tenía encuentros con los niños de la comunidad, y muy frecuentemente, ligado al uso del la grabadora. Por lo demás, este fenómeno se observa claramente en los programas de radio realizados por los mismos niños en el marco del proyecto de radio comunal Sisichakunaq pukllaynin de la Asociación Pukllasunchis, así como en una serie de videos realizados por los jóvenes de la comunidad de Cacchin (Lares) dentro del proyecto Amauta (CBC)13. De manera que en el momento de una presentación personal, después de anunciar nombre y apellido, así como el de sus padres, y en algunos casos de sus hermanos, los niños formulan una adivinanza o entonan una canción. Los términos utilizados pueden variar. Así, los niños podrán usar indistintamente los verbos, willa- «avisar» o ruwa- «hacer». De la misma manera, el acertijo es designado con el término quechua watuchi, con el préstamo del español «adivinanza», pero también con la fórmula misma de la pregunta imasmari:

13 Taller de Investigación/Creación Audiovisual en la comunidad de Ccachin, departamento de Cuzco, Perú, 2007, dirigido por el responsible del proyecto Amauta, Walter Angel Aparicio. 
Nuqaq sutiymi Vanessa Yuthu Mamani Cruz.

Me llamo ....

Huk watuchiykita ruwaramusaykichik

Te voy a hacer tu adivinanza

Imasmari, imasmari,

¿Qué cosa será, qué cosa será?

Hawan puna, ukhun yunqa

Por fuera puna y por dentro selva

imataq kanmanri?

¿qué cosa sería?

wachala14

mantilla

La dimensión colectiva de la realización de las adivinanzas hace de estas un caso ejemplar del carácter dialógico de los actos narrativos quechua, subrayados por Mannheim \& Van Vleet (2000)15. Efectivamente, las adivinanzas son esencialmente relatos dialogados, puesto que un niño propone un acertijo y los demás deben resolverlo.

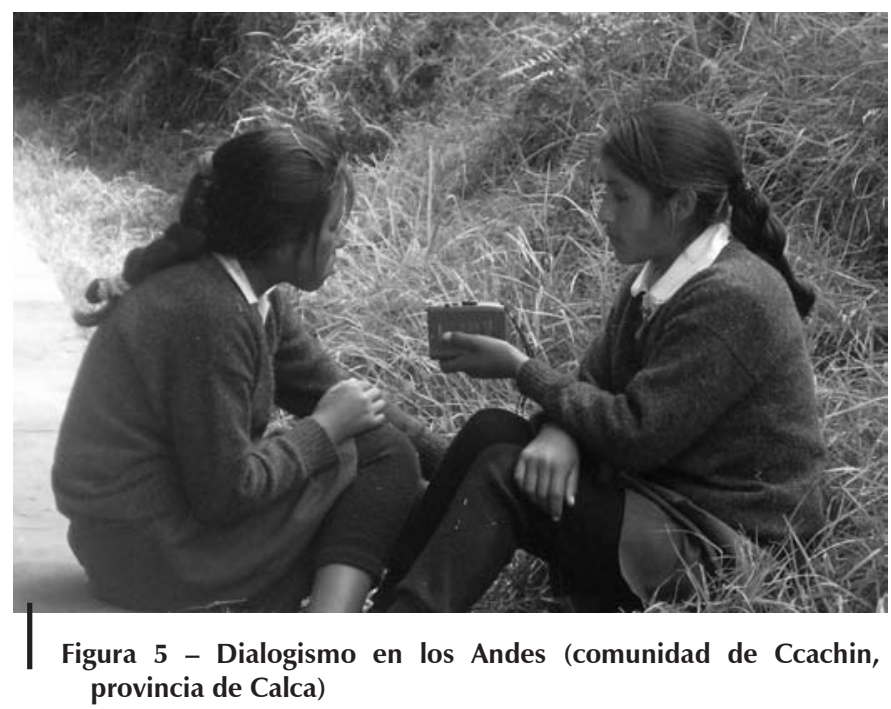

(C) Palmira La Riva González, 2011

14 La wachala es una mantilla que llevan las mujeres para guardar la coca y el dinero.

15 Sobre los diferentes niveles dialógicos de las interacciones orales en quechua ver Mannheim \& Van Vleet (2000). 
La función de socialización de las adivinanzas es explícitamente subrayada por Virginia Zavala en su estudio sobre la oralidad y escritura en educación intercultural bilingüe, señalando que las adivinanzas son introducidas en la escuela para «mejorar la socialización de los niños». Sin embargo, como advierte esta autora, las potencialidades y funciones pedagógicas de estas prácticas verbales son subestimadas, por no decir ignoradas por los maestros:

[...] en la mayoría de los casos, el maestro no discute, analiza, cuestiona o crea una adivinanza. Tampoco aprovecha este juego verbal como un recurso clave para el acto pedagógico. Su única función parece ser la de crear un buen ambiente (Zavala, 2001: 31)16.

En resumen, las adivinanzas junto a otras realizaciones lingüísticas, como las canciones o los insultos rituales al que participan los jóvenes y adultos durante el periodo de Carnaval, juegan un rol fundamental en el proceso continuo de socialización de niños y adultos.

\section{CONCLUSIÓN}

En la trama continua de la existencia que los ritos de paso ayudan a conformar, el ritual del rutuchikuy (corte de pelo), marca un momento esencial en el desarrollo cognitivo y social de los niños. Con este ritual asistimos al despliegue de las competencias lingüísticas estrechamente relacionadas con la fijación del animu al cuerpo, en el largo proceso de la construcción del cuerpo-persona. Desde la primera infancia, los niños se dedican a distintos géneros discursivos: cantos, cuentos y adivinanzas. La performance y realización de este último género contribuye al desarrollo cognitivo y social del niño por medio del desciframiento del lenguaje metafórico del mismo, y el contexto dialógico en el que se lleva a cabo. Es así que por medio de este juego verbal los niños se inician a distintos aspectos de su cultura. Digamos para concluir, que si esta socialización, a la cual las adivinanzas contribuyen de forma evidente, es en los Andes profundamente sexuada desde antes del nacimiento de los niños y niñas (La Riva González, 2010). En lo que concierne la performance de los juegos de lenguaje no tenemos todavía suficientes elementos empíricos que nos permitan establecer una distinción entre realizaciones lingüísticas diferenciadas por género. A primera vista, en el grupo de edad abordado no parecieran existir diferencias, estas aparecen de forma más explicita hacia la pubertad en la que los juegos de enigmas se relacionan con las prácticas sexuales y relaciones amorosas como lo consignan Isbell y Roncalla. La realización de los diversos juegos de lenguaje: adivinanzas, enigmas, insultos rituales y canciones acompañan el largo proceso de construcción del cuerpopersona y socialización de los individuos en los Andes entre realización individual y saberes colectivos.

16 No consideramos en este artículo el contexto de diglosia en el que se encuentran inmersas las prácticas discursivas locales o tradicionales. Para un análisis detallado de esta situación cf. Zavala, 2001; Arnold \& Yapita, 2000). 


\section{Referencias citadas}

ACADEMIA MAYOR DE LA LENGUA QUECHUA DE QOSQO, 2005 - Diccionario quechua-español-quechua, xxxi + 928 pp.; Cuzco: Municipalidad de Qosqo.

ALTAMIRANO RUA, T., 1984 - Watuchicuna (Adivinanzas quechuas). Anthropologica, 2 (2): 387-401.

ARÁOZ, D. \& SALAS, A., 1993 - Gramática quechua. Pautas fundamentales, 230 pp.; Cusco: Instituto de Pastoral Andina.

ARNOLD, D. Y. \& YAPITA, J. de D., 1996 - Los caminos de género en Qaqachaka: Saberes femeninos y discursos textuales alternativos en los Andes. In: Ser mujer indígena, chola o birlocha en la Bolivia postcolonial de los años 90 (S. Rivera Cusicanqui, ed.): 303-392; La Paz: Subsecretaría de Género/CID.

ARNOLD, D. Y. \& YAPITA, J. de D., 2000 - El rincón de las cabezas: luchas textuales, educación y tierras en los Andes, iii + 490 pp.; La Paz, Bolivia: UMSA e ILCA.

ARNOLD, D. Y., \& YAPITA, J. de D., 2002 - Las wawas del Inka: hacia la salud materna intercultural en algunas comunidades andinas, 275 pp.; La Paz, Bolivia: ILCA.

BAKHTINE, M. M., 1970 - Problèmes de la poétique de Dostoïevski, 316 pp.; Lausanne: L'Âge d'homme.

BASTIDE, R., 1970 - Le rire et les courts-circuits de la pensée. In: Échanges et communications: mélanges offerts à Claude Lévi-Strauss à l'occasion de son 60ème anniversaire: 953-963; París: The Hague, Mouton.

BERGONNIER-DUPUY, G., 2005 - L'enfant, acteur et-ou sujet au sein de la famille, 214 pp.; Ramonville Saint-Agne: Érès.

BOURDIEU, P., 1982 - Ce que parler veut dire: l'économie des échanges linguistiques, 243 pp.; París: Fayard.

CÁCERES VARGAS, G., 1996 - Riqsinakusun (conozcámonos), 77 pp.; Ediciones Kuyay.

CALAME-GRIAULE, G., 1963 - L'art de la parole dans la culture africaine. Présence Africaine, 47: 73-91.

CALAME-GRIAULE, G., 1970 - Pour une étude ethnolinguistique des littératures orales africaines. Langages, 5 (18): 22-47.

CERRÓN-PALOMINO, R., 1987 - Lingüística quechua, 426 pp.; Cusco: Centro de Estudios Rurales Andinos «Bartolomé de las Casas».

CHRISTINAT, J. L., 1989 - Des Parrains pour la vie : parenté rituelle dans une communauté des Andes péruviennes, 231 pp.; Neuchâtel, París: Institut d'Ethnologie, Éditions $\mathrm{MSH}$.

DELALANDE, J. (ed.), 2009 - Des enfants entre eux : Des jeux, des règles, des secrets, 153 pp.; París: Éditions Autrement.

Entrevista de Jean-Pierre Faye con Román Jakobson, 1970 - Hors Champs France Culture, 23 de julio.

FAÏK NZUJI, M., 1976 - Devinettes tonales = Tusumwinu, 92 pp.; París: SELAF.

FALLER, M., 2007 - The Cuzco Quechua Conjectural: an illocutionary analysis of an epistemic evidential. In: Deal, Amy Rose. UMOP 35: Proceedings of the 4th Conference on the Semantics of Underrepresented Languages of the Americas: The Semantics of Under-Represented Languages in the Americas; 24 May 2007-26 May 2007; Universidade de Sao Paulo. Amherst: GLSA; 2007.

FERNÁNDEZ JUÁREZ, G., 1995a - Imágenes, intelecto, palabras. El ojo del sueño en la formación de un «maestro» ceremonial aymara. Revista Andina, 13 (2): 389-420; Cusco: Centro de Estudios Regionales Andinos «Bartolomé de las Casas». 
Watuchi. Enigmas y saberes infantiles en los Andes del sur del Perú

FERNÁNDEZ JUÁREZ, G., 1995b - El banquete aymara: mesas y yatiris, 570 pp.; La Paz: Hisbol.

FERNÁNDEZ JUÁREZ, G., 1997 - Entre la repugnancia y la seducción: ofrendas complejas en los Andes del Sur, 250 pp.; Cusco: Centro de Estudios Regionales Andinos «Bartolomé de Las Casas».

FREUD, S., 1983 [1905] - Le mot d'esprit et ses rapports avec l'inconscient, 408 pp.; París: Gallimard.

GLASS-COFFIN, B., 2003 - La perspectiva de género en el curanderismo en el Norte del Perú: Metáforas, modelos y manifestaciones de la diferencia. In: Tradición popular: arte y religión de los pueblos del norte del Perú (L. Millones, H. Tomoeda \& T. Fujii, eds.): 67-94; Osaka, Japan: National Museum of Ethnology (Senri Ethnological Reports n. $\left.{ }^{\circ} 43\right)$.

GONZÁlEZ HOLGUÍN, D., 1952 [1608] - Vocabulario de la lengua general de todo el Perú Ilamada lengua qquichua o del Inca, xliv + 697 pp.; Lima: Universidad Nacional Mayor de San Marcos.

GUMPERZ, J. J. \& HYMES, D. H., 1986 [1972] - Directions in Sociolinguistics: The Ethnography of Communication, $x+598$ pp.; Oxford, Nueva York: B. Blackwell.

HAMNETT, I., 1967 - Ambiguity, Classification and Change: The Function of Riddles. Man, 2: 379-392.

HARRIS, O., 1980 - The Power of Signs: Gender, Culture and the Wild in the Bolivian Andes. In: Nature, Culture and Gender (C. MacCormack \& M. Strathern, eds.): 7095; Cambridge: Cambridge University Press.

HYMES, D. H., 1964 - Language in culture and society: a reader in linguistics and anthropology, xxv + 764 pp.; Nueva York: Harper \& Row.

ISBELL, B. J. \& RONCALLA, F., 1977 - The Ontogenesis of Metaphors: Riddle Games among Quechua Speakers Seen as Cognity Discovery Procedures. Journal of Latin American Lore, 3, 1: 19-49.

ITIER, C., 1997 - Parlons quechua : la langue du Cuzco, 207 pp.; París: Éditions L'Harmattan. ITIER, C., 2011 - Dictionnaire quéchua-français (1st ed.), 319 pp.; París: I'AsiathèqueMaison des langues du monde.

JAKOBSON, R., 1963 - Les fondations du langage, 260 pp.; París: Les Éditions de Minuit.

JAKOBSON, R., 1977 - Huit questions de poétique, 188 pp.; París: Éditions du Seuil.

KLEYMEYER, C. D., 1993 - Imashi! Imashi!: adivinanzas poéticas de los campesinos del mundo andino, Ecuador, Perú y Bolivia, 215 pp.; Quito: Editorial Abya Yala.

LA RIVA GONZÁLEZ, P., 2005 - Las representaciones del "animu" en los Andes del sur peruano. Revista andina, 41: 63-88.

LA RIVA GONZÁLEZ, P., 2010 - Rêves de fleurs et rêves de fruits : une construction andine du genre. Journal de la société des américanistes, 96 (96-2): 181-203.

LA RIVA GONZÁLEZ, P., 2012 - De l'enfant rêvé à I'enfant construit. Représentations de la petite enfance dans les Andes du Sud. In: Modèles d'enfances. Successions, transformations, croisements (D. Bonnet, C. Rollet \& C.-É. de Suremain, eds): 1937; París: Éditions des Archives Contemporaines.

LAKOFF, G. \& JOHNSON, M., 1985 - Les métaphores dans la vie quotidienne, 254 pp.; París: Éditions de Minuit.

LINDENFELD, J., 1984 - De l'Ethnographie de la communication à la sociolinguistique interactionniste. L'Homme, 24 (3): 131-135.

LIRA, J. A., 1982 - Diccionario Kkechuwa-Español, 345 pp.; Bogotá, Colombia: Secretaría Ejecutiva del Convenio Andrés Bello (2da Edición). 
MANNHEIM, B. \& VAN VLEET, K. E., 2000 - Surtout, ne vous endormez pas dans un bus : Le dialogisme dans la narration quechua méridionale. In: Les rituels du dialogue: promenades ethnolinguistiques en terres amérindiennes. (A. Monod-Becquelin \& P. Erikson, eds.): 29-78; Nanterre: Société d'ethnologie.

MURÚA, M. de., 1946 [1590] - Historia del origen y genealogía real de los reyes incas del Perú: Introducción, notas y arreglo por Constantino Bayle, xv + 444 pp.; Madrid: C. Bermejo Impresor.

NAVARRO DEL AGUILA, V., 1947 - Folklore nacional: calendario de fiestas populares del Departamento del Cusco. Trabajo de investigación presentado al concurso de la Cátedra de Folklore en la Universidad del Cusco.

POLIA MECONI, M., 1994 - Cuando Dios lo permite: Encantos y arte curanderil; llas estructuras culturales de la medicina tradicional andina], 202 pp.; Lima: Editorial Prometeo.

RABAIN-JAMIN, J., 2003 - Enfance, âge et développement chez les Wolof du Sénégal. L'Homme. Revue française d'anthropologie, 167-168: 49-66.

RICARD LANATA, X., 2010 - Les voleurs d'ombre : l'univers religieux des bergers de I'Ausangate, Andes centrales, 540 pp.; Nanterre: Société d'ethnologie.

ROBERTS, J. \& FOREMAN, M. L., 1986 [1972] - Riddles: expressive models of interrogations. In: Directions in Sociolinguistics: The Ethnography of Communication (J. J. Gumperz \& D. H. Hymes, eds.): 180-209; Oxford, Nueva York: B. Blackwell.

SHERZER, J. \& DARNELL, R., 1986 [1972] - Outline guide for the ethnographic study of speech use. In: Directions in Sociolinguistics: The Ethnography of Communication (J. J. Gumperz \& D. H. Hymes, eds.): 548-554; Oxford, Nueva York: B. Blackwell.

SIROTA, R., 2006 - Éléments pour une sociologie de l'enfance, 325 pp.; Rennes: Presses universitaires de Rennes.

SIROTA, R., 2012 - L'enfance au regard de sciences sociales. AnthropoChildren, 1. http://popups.ulg.ac.be/AnthropoChildren/document.php?id=921

SUREMAIN, C.-E. de, 2010 - Quand « le cheveu fait l'homme ». Autrepart, 55 (3): 125139.

TODOROV, T., 1978 - La devinette. In: Les genres du discours: 223-245; París: Seuil.

TODOROV, T., 1981 [1926] - Mikhaïl Bakhtine : le principe dialogique, suivi de: ecrits du cercle de Bakhtine, 318 pp.; París: Éditions du Seuil.

VOLOCHINOV/BAKHTINE, véase TODOROV, 1981.

WILLETT, T., 1988 - A cross-linguistic survey of the grammaticalization of evidentiality. Studies in Langage, 12: 51-97.

WILLIAMS, T. R., 1963 - The Form and Function of Tambunan Dusun Riddles. Journal of American Folklore, 76: 95-110.

WITTGENSTEIN, L., 2005 - Recherches philosophiques, 367 pp.; París: Éditions Gallimard.

ZAVALA, V., 2001 - Oralidad y escritura en la educación bilingüe (a propósito de interculturalidad), 50 pp.; Lima: Plancad-Gtz-Kfw.

ZAVALA, V., 2006 - La oralidad como performance: un análisis de géneros discursivos andinos desde una perspectiva sociolingüística. Boletín del Instituto Riva-Agüero, 33: 129-137. 